\title{
Effects of multi-pion correlations on the source distribution in ultra-relativistic heavy-ion collisions
}

\author{
Q.H. Zhang \\ Institut für Theoretische Physik, Universität Regensburg, D-93040 Regensburg, Germany
}

\begin{abstract}
Multi-pion correlation effect on the source distribution is studied. It is shown that multi-pion Bose-Einstein correlation make the average radius of the pion source become smaller. The isospin effect on the pion multiplicity distribution and the source distribution is also discussed.
\end{abstract}

PACS number(s): $11.38 \mathrm{Mh}, 11.30 \mathrm{Rd}, 25.75 \mathrm{Dw}$

Ultrarelativistic hadronic and nuclear collisions provide a unique environment to create dozens, and in some cases hundreds, of pions [1 1 [3]. To study the pion source distributions in these processes, therefore, one must take into account the effects of multi-pion Bose-Einstein (BE) correlations [1] [14]. Among others, Lam and Lo [4] suggested that the larger isospin fluctuations were due to the Bose nature of the emitted pions. Pratt [7] suggested that if the number of bosons in a unit value of phase space is large enough, bosons may condense into the same quantum state and a pion laser could be created. Considering isospin effect, Pratt and Zelevinsky [8] used the method in Ref. [7] to explain Centauro events [15,16]. Zajc [6] first used Monte-Carlo method to analyse multi-pion Bose-Einstein correlation effects on two-pion interferometry. Detail derivation of multi-pion Bose-Einstein correlation can be found in Ref. [12]. The bosonic nature and isospin of the pion should affect the single pion spectrum distribution in coordinate space. However, this issue has not yet been discussed in the literature. The purpose of this paper is to analyse the effects of multi-pion correlation and isospin on the source distribution in coordinate space.

We begin with write down the definition for the $n$-pion inclusive source distribution: 


$$
P_{n}\left(x_{1}, \cdots, x_{n}\right)=\left\langle j^{*}\left(x_{1}\right) \cdots j^{*}\left(x_{n}\right) j\left(x_{1}\right) \cdots j\left(x_{n}\right)\right\rangle
$$

which can be explained as the probability of observing $n$ pions at point $\left\{x_{i}, i=1, n\right\}$ all in the same $n$ pion event. Here $j(x)$ is the current of pions, which can be expressed as 9 , 12, 13.

$$
j(x)=\int j\left(x^{\prime}, p\right) \exp \left\{i p\left(x-x^{\prime}\right)\right\} \gamma\left(x^{\prime}\right) d^{4} x^{\prime} d^{4} p,
$$

where $j\left(x^{\prime}, p\right)$ is the probability amplitude of finding a pion with momentum $p$, emitted by the emitter at $x^{\prime} \cdot \gamma\left(x^{\prime}\right)$ is a random phase factor which has been taken away from $j\left(x^{\prime}, p\right)$. All emitters are uncorrelated in coordinate space if assuming:

$$
\left\langle\gamma^{*}(x) \gamma(y)\right\rangle=\delta^{4}(x-y)
$$

Taking the phase average and using eq. (3), one can re-express the $n$-pion inclusive distribution (eq.(1)) as

$$
P_{n}\left(x_{1}, \cdots, x_{n}\right)=\sum_{\sigma} \rho_{1, \sigma(1)} \cdots \rho_{n, \sigma(n)},
$$

with

$$
\rho_{i, j}=\rho\left(x_{i}, x_{j}\right)=<j^{*}\left(x_{i}\right) j\left(x_{j}\right)>, \quad \int \rho(x, x) d^{4} x=n_{0} .
$$

Here $\sigma(i)$ denotes the $i$ th element of a permutation of the sequence $1,2,3, \cdots, n$, and the sum over $\sigma$ runs over all $n$ ! permutations of this sequence. $n_{0}$ is the mean pion multiplicity without multi-pion Bose-Einstein correlations.

Taking into account the $n$-pion correlation effect, the normalized modified $i$-pion inclusive distribution in $n$ pion events, $P_{i}^{n}\left(x_{1}, \cdots, x_{i}\right)$, can be written as

$$
P_{i}^{n}\left(x_{1}, \cdots, x_{i}\right)=\frac{\int \prod_{j=i+1}^{n} d^{4} x_{j} P_{n}\left(x_{1}, \cdots, x_{n}\right)}{\int \prod_{j=1}^{n} d^{4} x_{j} P_{n}\left(x_{1}, \cdots, x_{n}\right)},
$$

which can be explained as the probability of finding $i$ pions at point $\left\{x_{j}, j=1, i\right\}$ in $n$ pion events.

Now we define the function $G_{i}(x, y)$ as [0, 12] 


$$
G_{i}(x, y)=\int \rho\left(x, x_{1}\right) d x_{1} \rho\left(x_{1}, x_{2}\right) d x_{2} \cdots \rho\left(x_{i-2}, x_{i-1}\right) d x_{i-1} \rho\left(x_{i-1}, y\right) .
$$

From the expression of $P_{n}\left(x_{1}, \cdots, x_{n}\right)$ (eq.(4)), the single pion inclusive distribution in coordinate space can be expressed as

$$
P_{1}^{n}(x)=\frac{1}{n} \frac{1}{\omega(n)} \sum_{i=1}^{n} G_{i}(x, x) \cdot \omega(n-i),
$$

with

$$
\omega(n)=\frac{1}{n !} \int P_{n}\left(x_{1}, \cdots, x_{n}\right) \prod_{k=1}^{n} d^{4} x_{k} .
$$

Here $\omega(n)$ is the pion multiplicity distribution probability. Experimentally, one usually mixes all events to analyse the single-pion inclusive distribution. Thus the single-pion inclusive distribution reads

$$
P_{1}^{\phi}(x)=\frac{\sum_{n=1}^{\infty} \omega(n) \cdot n \cdot P_{1}^{n}(x)}{<n>\sum_{n} \omega(n)},
$$

with

$$
<n>=\frac{\sum_{n=1}^{\infty} \omega(n) \cdot n}{\sum_{n} \omega(n)}
$$

Using eq.(8), one can writes down the single-pion inclusive distribution

$$
P_{1}^{\phi}(x)=\frac{1}{<n>} \sum_{i=1}^{\infty} G_{i}(x, x) .
$$

In the following, we will employ a simple model to analyse the multi-pion correlation effects on the source distribution. We assume that

$$
\rho(x, y)=n_{0} \cdot\left(\frac{1}{\pi R_{1}^{2}}\right)^{3 / 2} \exp \left(-(\vec{x}+\vec{y})^{2} / 4 R_{1}^{2}\right) \exp \left(-(\vec{x}-\vec{y})^{2} / 4 R_{2}^{2}\right) \sqrt{\delta\left(x_{0}\right) \delta\left(y_{0}\right)} .
$$

Here $R_{1}$ and $R_{2}$ are the parameters that represents the radius of the chaotic source and the correlation length of pions respectively. $x=\left(x_{0}, \vec{x}\right)$ are the pion's four dimensional coordinate. Then, $G_{n}(x, y)$ can be expressed as

$$
G_{n}(x, y)=n_{0}^{n} \cdot \sqrt{\delta\left(x_{0}\right) \delta\left(y_{0}\right)} \cdot \alpha_{n} \cdot \exp \left\{-a_{n} \cdot\left(\vec{x}^{2}+\vec{y}^{2}\right)+g_{n} \cdot \vec{x} \cdot \vec{y}\right\}
$$


where

$$
a_{n+1}=a_{1}-\frac{g_{1}^{2}}{4 b_{n}}, g_{n+1}=\frac{g_{1} \cdot g_{n}}{2 b_{n}}, b_{n}=a_{n}+a_{1},
$$

and

$$
\alpha_{n+1}=\alpha_{n} \cdot\left(\frac{1}{b_{n}}\right)^{3 / 2} \cdot\left(\frac{1}{R_{1}^{2}}\right)^{3 / 2}
$$

with

$$
a_{1}=\frac{1}{4 R_{1}^{2}}+\frac{1}{4 R_{2}^{2}}, g_{1}=\frac{1}{2 R_{2}^{2}}-\frac{1}{2 R_{1}^{2}}, \alpha_{1}=\left(\frac{1}{\pi R_{1}^{2}}\right)^{3 / 2}
$$

From the above formula, we obtain the pion source distribution in $n$ pion events $\left(P_{1}^{n}(\vec{x})=\right.$ $\left.\int P_{1}^{n}(x) d x_{0}\right)$, which we show in Fig. 1. Evidently, due to the multi-pion Bose-Einstein correlation effects, the pions are concentrated in the same state. The mean radius of the source becomes smaller. The larger the pion multiplicity, the larger the BE correlation effects on the source distribution. Multi-pion correlation effects on the source distribution $\left(P_{1}^{\phi}(\vec{x})=\int P_{1}^{\phi}(x) d x_{0}\right)$ is shown in fig.2, where we mix the pion multiplicity. It is clear that as the mean pion multiplicity becomes larger, the multi-pion correlation effects on the source distribution become larger.

The pion state with isospin can be written as [10,17

$$
\left|\phi>=\exp \left(\int \vec{j}(x) \cdot \vec{a}^{+}(x)\right)\right| 0>
$$

where $\vec{j}$ and $\vec{a}$ are the vectors in isospin space which can be expressed as

$$
\vec{j}(x)=j(x)\left[\frac{1}{\sqrt{2}} \sin \theta e^{-i \phi}, \cos \theta,-\frac{1}{\sqrt{2}} \sin (\theta) e^{i \phi}\right],
$$

and

$$
\vec{a}^{+}(x)=\left[a_{\pi^{-}}^{+}(x), a_{\pi^{0}}^{+}, a_{\pi^{+}}^{+}\right]
$$

Then the state with total isospin $I$ and $z$ component of isospin $I_{z}$ is

$$
\left|\phi, I, I_{z}>=\int \sin \theta d \theta d \phi Y_{I, I_{z}}^{*}(\theta, \phi) \exp \left(\int \vec{j}(x) \cdot \vec{a}^{+}(x)\right)\right| 0>.
$$


$Y_{I, I_{z}}(\theta, \phi)$ is the spherical harmonic of angular momentum $I$ and $z$ component $I_{z}$. From the above formula we can calculate isospin effect on pion probability and pion source distribution. For the sake of simplicity, we will only consider in the following the $I=0, I_{z}=0$ case. One can easily check that $\mid \phi, 0,0>$ can be expanded in Fock space as

$$
\left|\phi, 0,0>=\sum_{n_{\pi^{0}}} \sum_{n_{c} / 2} \frac{1}{2^{n_{c} / 2}} B\left[\frac{1}{2}+\frac{n_{\pi^{0}}}{2}, \frac{n_{c}}{2}+1\right]\right| n_{\pi^{0}}>\left|\frac{n_{c}}{2}>\right| \frac{n_{c}}{2}>,
$$

with

$$
\left|n_{\pi^{0}, \pi^{+}, \pi^{-}}>=\frac{\left(\int j(x) a_{\pi^{0}, \pi^{+}, \pi^{-}}^{+} d^{4} x\right)^{n}}{n !}\right| 0>.
$$

Here $\left|n_{\pi}\right\rangle$ denote $n$ pion state and $B(x, y)$ is the Beta function. $n_{c}=n_{\pi^{+}}+n_{\pi^{-}}$is the total number of charged pions. Due to the isospin conservation, we have $n_{\pi^{+}}=n_{\pi^{-}}=\frac{n_{c}}{2}$ and $n_{\pi^{0}}$ must be even. At this stage we can calculate $\pi^{0}$ and $\pi^{+,-}$probability distribution according to

$$
P\left(n_{\pi^{0}}\right)=\frac{\sum_{n_{c}} \omega\left(n_{\pi^{0}}\right) \frac{1}{2^{n_{c}}} B^{2}\left(\frac{n_{\pi^{0}}+1}{2}, \frac{n_{c}}{2}+1\right) \omega^{2}\left(\frac{n_{c}}{2}\right)}{\sum_{n_{\pi^{0}}} \sum_{n_{c}} \omega\left(n_{\pi^{0}}\right) \frac{1}{2^{n_{c}}} B^{2}\left(\frac{n_{\pi^{0}}+1}{2}, \frac{n_{c}}{2}+1\right) \omega^{2}\left(\frac{n_{c}}{2}\right)},
$$

and

$$
P\left(n_{\pi^{+}}=n_{\pi^{-}}=\frac{n_{c}}{2}\right)=\frac{\sum_{n_{\pi^{0}}} \omega\left(n_{\pi^{0}}\right) \frac{1}{2^{n_{c}}} B^{2}\left(\frac{n_{\pi^{0}+1}}{2}, \frac{n_{c}}{2}+1\right) \omega^{2}\left(\frac{n_{c}}{2}\right)}{\sum_{n_{\pi^{0}}} \sum_{n_{c}} \omega\left(n_{\pi^{0}}\right) \frac{1}{2^{n_{c}}} B^{2}\left(\frac{n_{\pi^{0}}+1}{2}, \frac{n_{c}}{2}+1\right) \omega^{2}\left(\frac{n_{c}}{2}\right)} .
$$

The $\pi^{0}$ and $\pi^{+,-}$probability distributions are shown in Fig.3. For comparison, the pion probability without isospin conservation is also shown as solid line in Fig.3. We notice that due to isospin effect, the $\pi^{0}$ and $\pi^{+,-}$probability distributions are quite different from each other now. This phenomena have ever been noticed in Ref. [8]. But the method given here enable us to calculated the isospin effect for any isospin state, not limited to iso-singlet state as discussed in Ref. [8]. From the $\pi^{0}$ and $\pi^{+,-}$probability distributions, we acquire the mean multiplicities $<n_{\pi^{0}}>$ and $<n_{\pi^{+,-}}>$

$$
<n_{\pi^{0}}>=\frac{\sum_{n_{\pi^{0}}} \sum_{n_{c}} \frac{1}{2^{n_{c}}} B^{2}\left(\frac{n_{\pi^{0}}}{2}+\frac{1}{2}, \frac{n_{c}}{2}+1\right) \omega^{2}\left(\frac{n_{c}}{2}\right) n_{\pi^{0}} \omega\left(n_{\pi^{0}}\right)}{\sum_{n_{\pi^{0}}} \sum_{n_{c}} \frac{1}{2^{n_{c}}} B^{2}\left(\frac{n_{0}}{2}+\frac{1}{2}, \frac{n_{c}}{2}+1\right) \omega^{2}\left(\frac{n_{c}}{2}\right) \omega\left(n_{\pi^{0}}\right)},
$$

and 


$$
<n_{\pi^{+,-}}>=\frac{\sum_{n_{\pi^{0}}} \sum_{n_{c}} \frac{1}{2^{n_{c}}} B^{2}\left(\frac{n_{\pi^{0}}}{2}+\frac{1}{2}, \frac{n_{c}}{2}+1\right) \omega^{2}\left(\frac{n_{c}}{2}\right) n_{\pi^{+},-} \omega\left(n_{\pi^{0}}\right)}{\sum_{n_{\pi^{0}}} \sum_{n_{c}} \frac{1}{2^{n_{c}}} B^{2}\left(\frac{n_{0}}{2}+\frac{1}{2}, \frac{n_{c}}{2}+1\right) \omega^{2}\left(\frac{n_{c}}{2}\right) \omega\left(n_{\pi^{0}}\right)} .
$$

It is well known that in the case of the isospin singlet, if the multi-particle BE correlation in coordinate space is neglected $\left(R_{2}=0 \mathrm{fm}\right)$, there would be $<n_{\pi^{0}}>=<n_{\pi^{+}}>=<n_{\pi^{-}}>$ [17]. As the multi-particle BE correlation is included in coordinate space, however, such a simple relation does not hold for the isosinglet any more. As a example, to make a quantitative sense, we set $R_{1}=5 \mathrm{fm}, R_{2}=0.8 \mathrm{fm}$ and $n_{0}=20$ and obtain $n_{\pi^{0}}=2.86$ and $n_{\pi^{+}}=n_{\pi^{-}}=9.38$.

The $\pi^{0}$ and $\pi^{+,-}$source distribution reads

$$
\begin{aligned}
P_{\pi^{0}}(x) & =\frac{<\phi, 0,0\left|a_{\pi^{0}}^{+}(x) a_{\pi^{0}}(x)\right| \phi, 0,0>}{<\phi, 0,0 \mid \phi, 0,0><n_{\pi^{0}}>} \\
& =\frac{1}{<n_{\pi^{0}}>} \frac{\sum_{n_{\pi^{0}}} \sum_{n_{c}} \frac{1}{2^{n_{c}}} B^{2}\left(\frac{n_{\pi^{0}}}{2}+\frac{1}{2}, \frac{n_{c}}{2}+1\right) \omega^{2}\left(\frac{n_{c}}{2}\right) \sum_{i=1}^{n_{\pi^{0}}} G_{i}(x, x) \omega\left(n_{\pi^{0}}-i\right)}{\sum_{n_{\pi^{0}}} \sum_{n_{c}} \frac{1}{2^{n_{c}}} B^{2}\left(\frac{n_{\pi^{0}}}{2}+\frac{1}{2}, \frac{n_{c}}{2}+1\right) \omega^{2}\left(\frac{n_{c}}{2}\right) \omega\left(n_{\pi^{0}}\right)},
\end{aligned}
$$

and

$$
\begin{aligned}
P_{\pi^{+,-}}(x) & =\frac{<\phi, 0,0\left|a_{\pi^{+,-}}^{+}(x) a_{\pi^{+,-}}(x)\right| \phi, 0,0>}{<\phi, 0,0 \mid \phi, 0,0><n_{\pi^{+,-}}>} \\
& =\frac{1}{<n_{\pi^{+,-}}>} \frac{\sum_{n_{\pi^{0}}} \sum_{n_{c}} \frac{1}{2^{n_{c}}} B^{2}\left(\frac{n_{\pi^{0}}}{2}+\frac{1}{2}, \frac{n_{c}}{2}+1\right) \omega\left(n_{\pi^{0}}\right) \omega\left(\frac{n_{c}}{2}\right) \sum_{i=1}^{n_{\pi^{+},-}} G_{i}(x, x) \omega\left(\frac{n_{c}}{2}-i\right)}{\sum_{n_{\pi^{0}}} \sum_{n_{c}} \frac{1}{2^{n_{c}}} B^{2}\left(\frac{n_{\pi^{0}}}{2}+\frac{1}{2}, \frac{n_{c}}{2}+1\right) \omega^{2}\left(\frac{n_{c}}{2}\right) \omega\left(n_{\pi^{0}}\right)} .
\end{aligned}
$$

The $\pi^{0}$ and $\pi^{+,-}$inclusive distributions are shown in Fig.4. For comparison, we also give the $\pi$ inclusive distribution without isospin effect in the same figure. Notice that there are a difference among $\pi^{0}, \pi^{+,-}$and $\pi$ inclusive distribution caused by multi-particle BE correlations in coordinate space.

In this paper, we have not considered the energy constraint effects on the source distribution as done in Ref. [13. Also the source model present here is not a realistic model. As stressed in Ref. [6],8], for a more or less real model, the amount of calculational work will increase astronomically. To our best knowledge, there is so far no method which enables us to calculate quickly multi-particle BE correlation in the above process. But we think that for the purpose of illustrating the general features of multi-pion correlation effects on the source distribution, our toy model is good enough. In Ref. [18], Fowler et al. found that the 
restriction on the multiplicity have great influence on BE correlation. We did not address this issue, simply because our prime purpose on the paper is to discuss the multi-pion BE correlation and isospin effects on the source distribution in coordinate space, which has not yet been discussed in the previous publication. We leave the systematical discussion of all these factors on the source distribution for the future publication.

In conclusion, the multi-pion Bose-Einstein correlation effects on the pion source distribution has been discussed. We showed that multi-pion correlation make the average radius of the source become smaller. The larger the pion multiplicity, the larger the multi-pion correlation effects on the source distribution. Isospin effects on pion probability and pion source distribution are also discussed. We observed that multi-pion correlations distort the relation $<n_{\pi^{0}}>=<n_{\pi^{+}}>=<n_{\pi^{-}}>$which exists in isospin-singlet state without multi-particle BE correlation in coordinate space.

\section{Acknowledgement}

The author thanks Dr. Y. Pang for helpful discussions and to Dr. W. Lu for reading the

manuscript. This work was partly supported by the Alexander von Humboldt foundation in Germany. 


\section{REFERENCES}

[1] OPAL Collab. P.D. Acton et al., Phys. Lett. B 320417 (1994).

[2] NA35 Collab.. T. J. Humanic, Z. Phys. C38 79 (1988).

[3] E802 Collab.., T. Abbott et al., Nucl. Phys. A544 237 (1992).

[4] C.S. Lam and S.Y. Lo, Phys. Rev. Lett. 52,1184 (1984), Phys. Rev. D33 1336 (1986).

[5] W. Willis and C. Chasman, Nucl. Phys. A418 425c (1984).

[6] W. A. Zajc, Phys. Rev. D35 3396 (1987).

[7] S. Pratt, Phys. Lett. B301 159 (1993).

[8] S. Pratt and V. Zelevinsky, Phys. Rev. Lett 72, 816 (1994),

S. Pratt, in Quark-Gluon-Plasma 2, ed. R.C. Hwa (world Scientific Pub. Co. Singapore 1995)P700.

[9] S. Padula, M. Gyulassy, and S. Gavin, Nucl. Phys. B329 357 (1990).

[10] I.V. Andreev, M. Plümer and R.M. Weiner, Int. J. Mod. Phys. A8, 4577 (1993).

[11] J. Zimanyi and T.Csorgo, hep-ph/9705432

[12] W.Q. Chao, C.S. Gao and Q. H. Zhang, J. Phys. G21 847 (1995).

[13] Q.H. Zhang,W.Q. Chao and C.S. Gao, Phys. Rev. C52 2064 (1995).

[14] Q.H. Zhang, Phys. Lett. B406,366 (1997).

[15] C.M.G. Lattes,Y. Fujimoto, and S. Hasegewa, Phys. Rep. 65, 151 (1980).

[16] I.V. Andreev,M. Biyajima,V.A.Nechitailo, and N. Suzuki, Phys. Lett. B388,197 (1996) and reference cited there.

[17] D.Horn and R. Silver, Ann. Phys. (N.Y.)66, 509 (1971). 
[18] G.N. Fowler, E.M. Friedlander,X.C. He, C.C. Shin and R.M. Weiner, Phys. Lett. B253, 421 (1991).

\section{Figure Captions}

1. Multi-pion correlation effects on the source distribution. The solid line corresponds to the input source distribution. The dashed and dotted lines corresponds to $n=20,80$ respectively. The input value of $R_{1}$ and $R_{2}$ is $5 \mathrm{fm}$ and $0.8 \mathrm{fm}$ respectively.

2. Multi-pion correlation effects on the source distribution. The solid line corresponds to the input source distribution. The dashed and dotted lines corresponds to $\langle n\rangle=$ 22, 126 respectively. The input value of $R_{1}$ and $R_{2}$ is $5 \mathrm{fm}$ and $0.8 \mathrm{fm}$ respectively.

3. Pion multiplicity distribution. The solid line corresponds to the pion probability distribution without isospin effect. The dashed and dotted lines corresponds to $\pi^{0}$ and $\pi^{+,-}$probability distribution respectively. The input value of $R_{1}, R_{2}$ and $n_{0}$ is $5 \mathrm{fm}$, $0.8 \mathrm{fm}$ and 20 respectively.

4. Multi-pion correlation and isospin effects on the source distribution. The solid line corresponds to the source distribution without isospin effect. The dashed and solid lines corresponds to $\pi^{0}$ and $\pi^{+,-}$source distribution respectively. The input value of $R_{1}, R_{2}$ and $n_{0}$ is $5 \mathrm{fm}, 0.8 \mathrm{fm}$ and 40 respectively. 


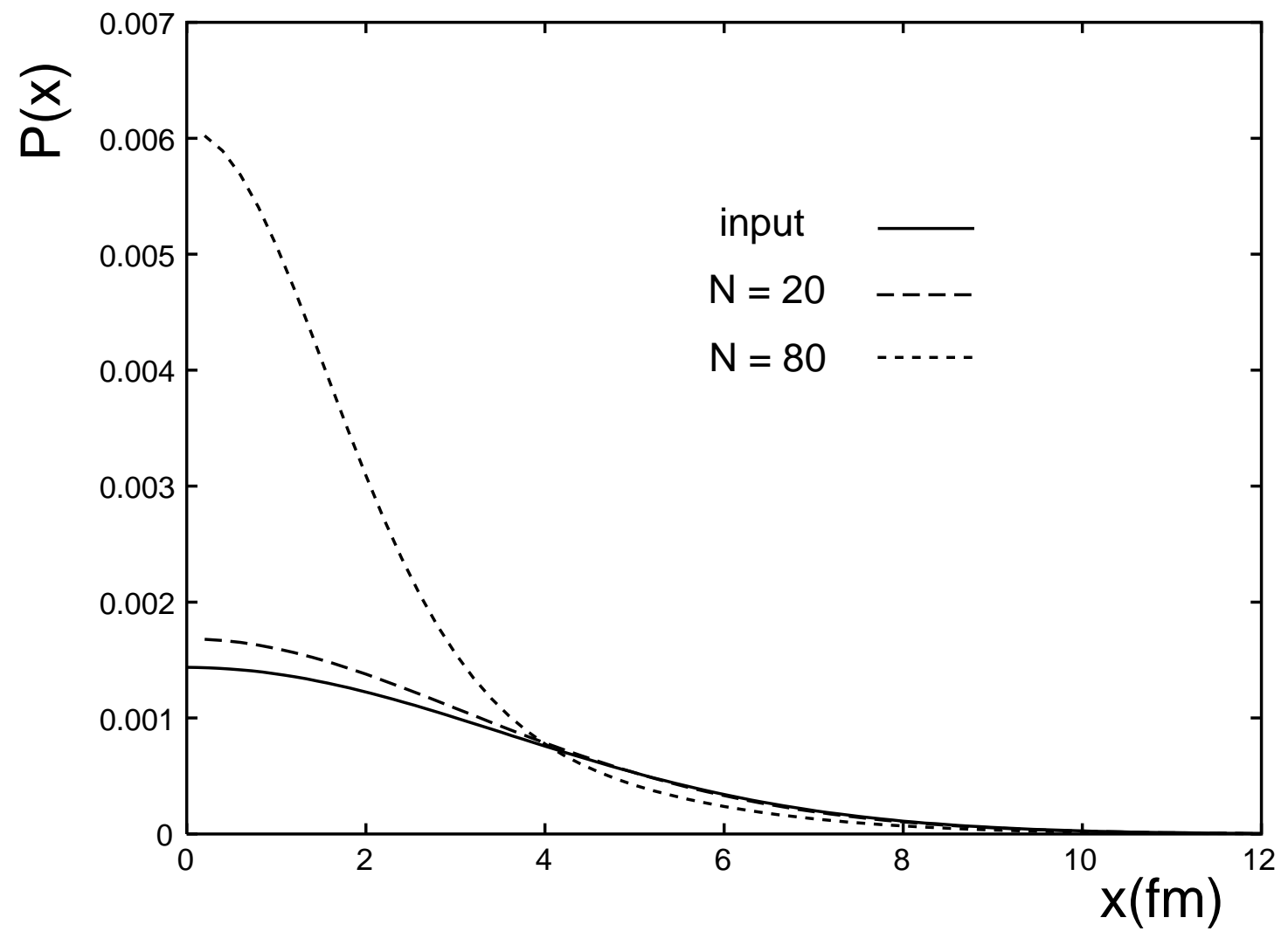

Fig.1 


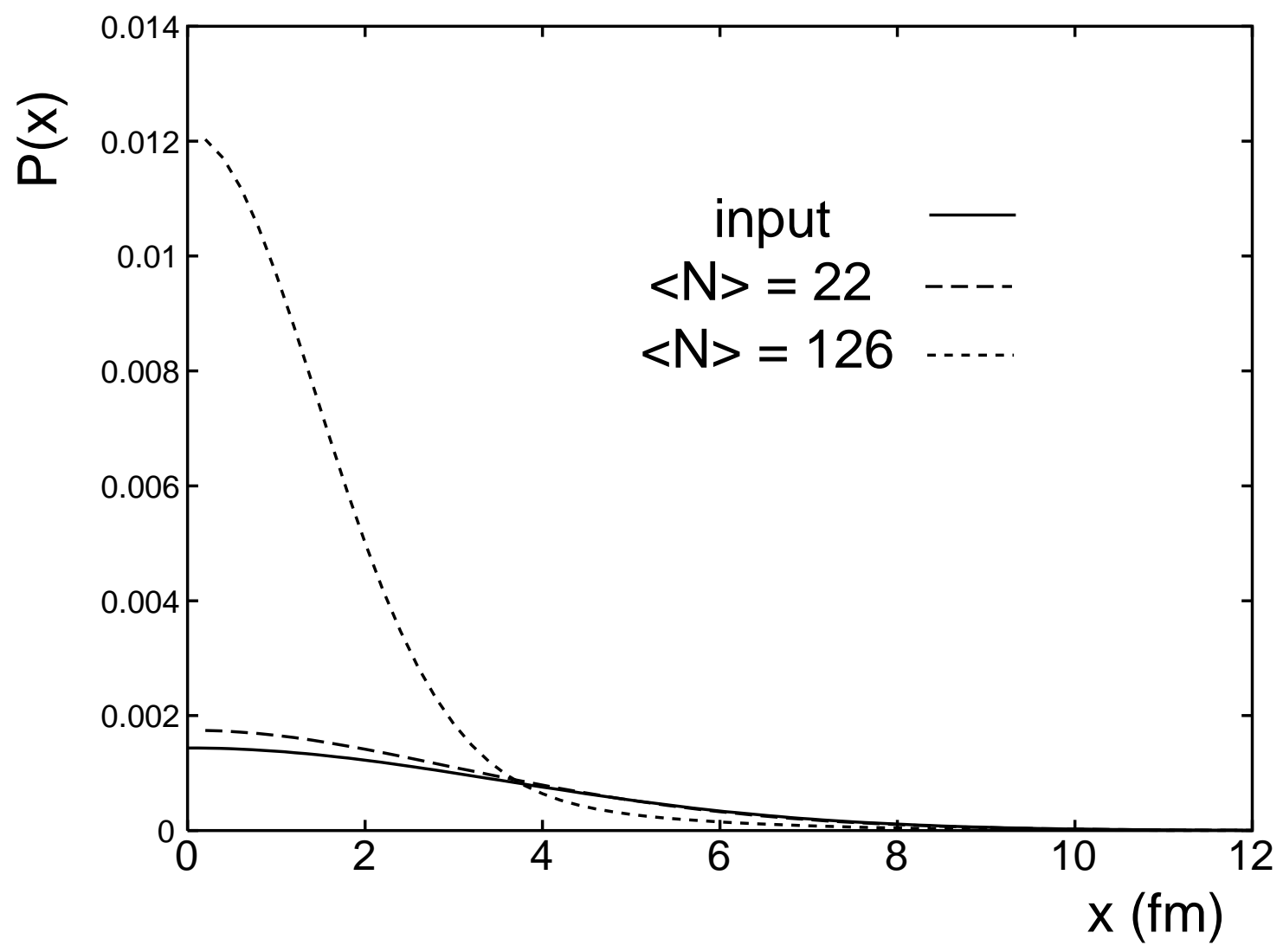

Fig.2 


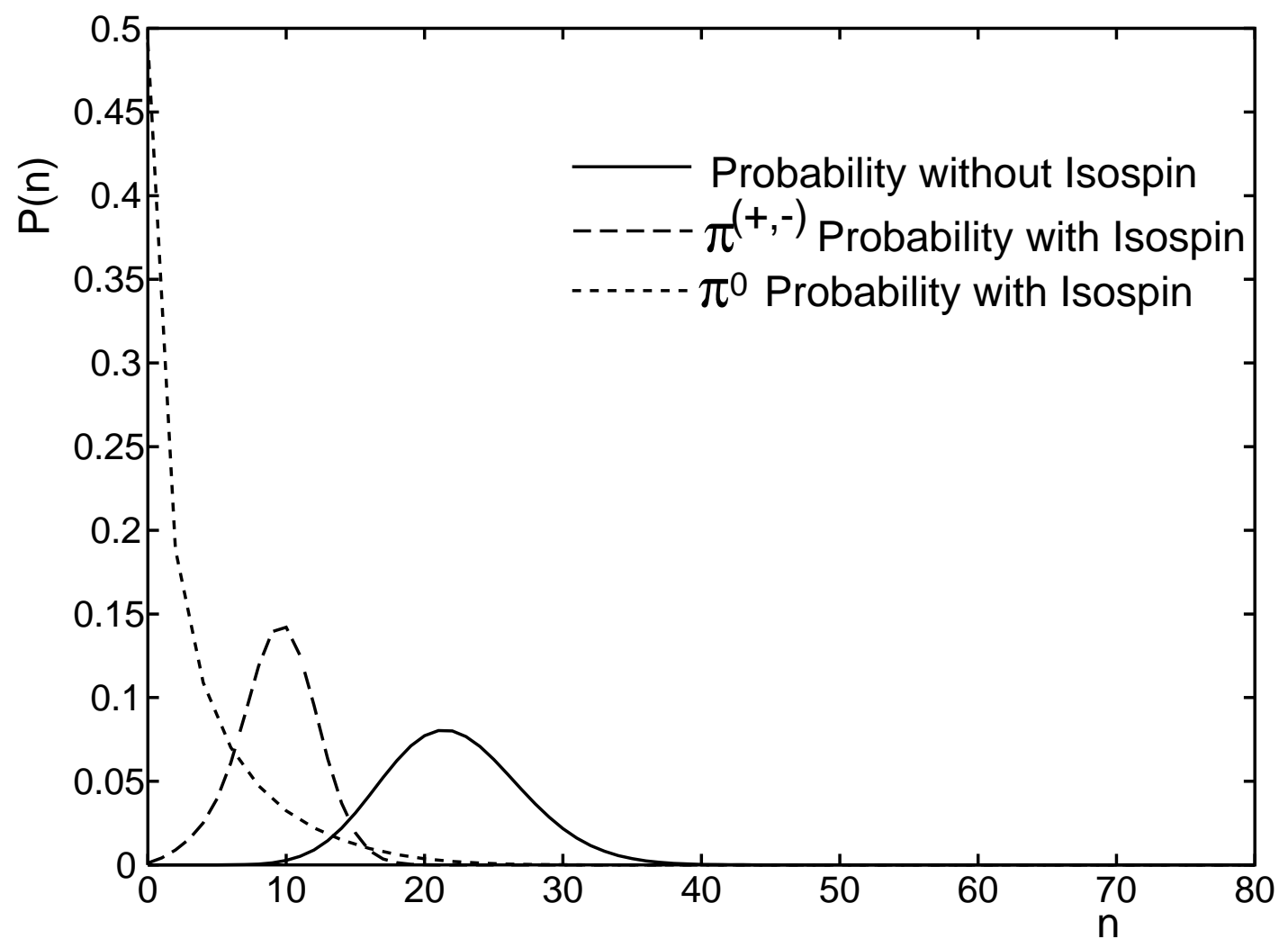

Fig.3 


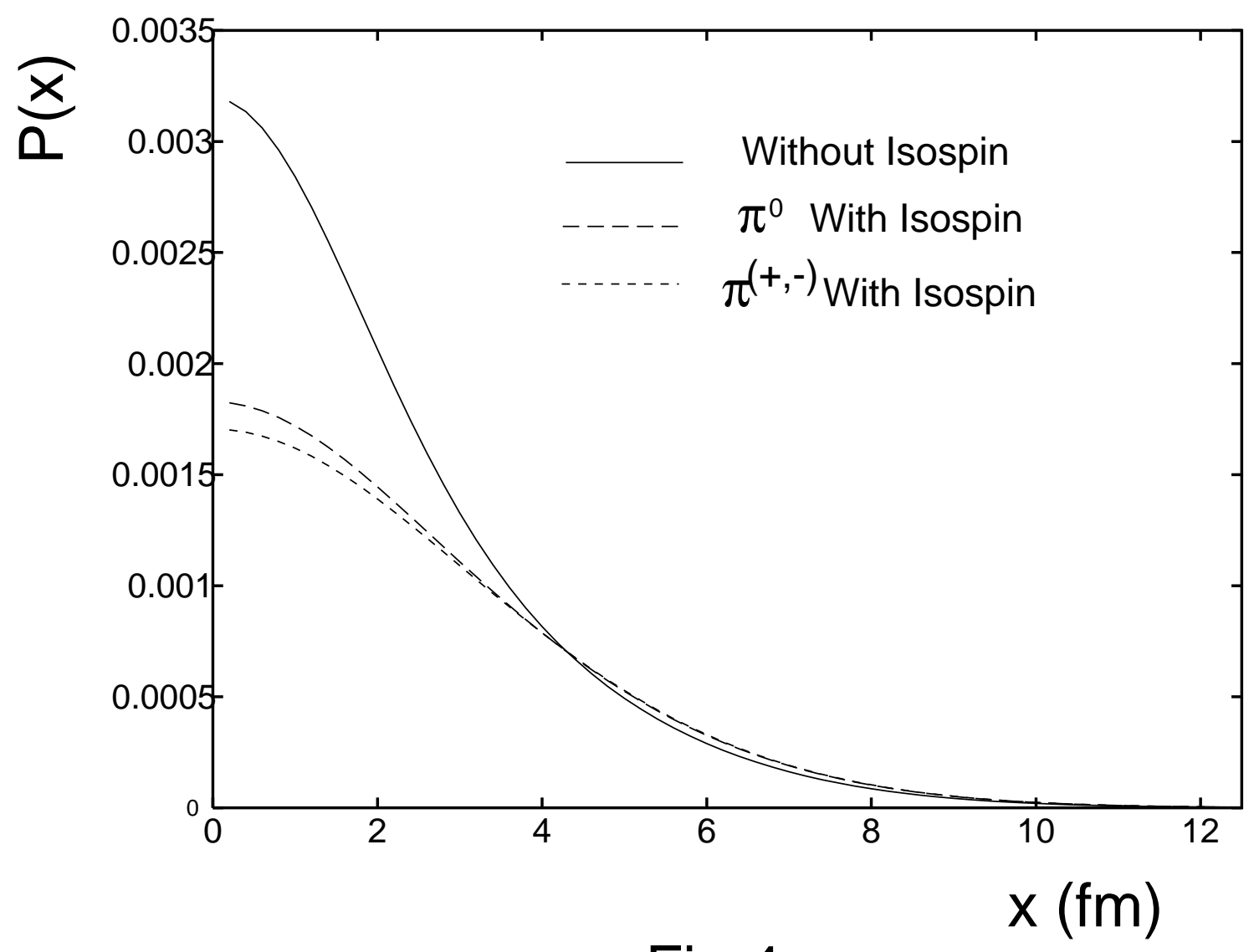

Fig.4 